\section{(6) OPEN ACCESS}

\title{
Immune activation by DNA damage predicts response to chemotherapy and survival in oesophageal adenocarcinoma
}

\author{
Richard C Turkington, ${ }^{1}$ Laura A Knight, ${ }^{2}$ Jaine K Blayney, ${ }_{1}^{1}$ Maria Secrier, ${ }^{3}$ \\ Rosalie Douglas, ${ }^{1}$ Eileen E Parkes, ${ }^{1}$ Eilis K Sutton, ${ }^{1}$ Leanne Stevenson, ${ }^{1}$ \\ Damian McManus, ${ }^{4}$ Sophia Halliday, ${ }^{1}$ Andrena M McCavigan, ${ }^{2}$ Gemma E Logan, ${ }^{2}$ \\ Steven M Walker, ${ }^{2}$ Christopher I Steele, ${ }^{2}$ Juliane Perner, ${ }^{5}$ Jan Bornschein, ${ }^{6}$ \\ Shona MacRae, ${ }^{7}$ Ahmad Miremadi, ${ }^{8}$ Eamon McCarron, ${ }^{4}$ Stephen McQuaid, ${ }^{9}$ \\ Kenneth Arthur, ${ }^{9}$ Jacqueline A James, ${ }^{9}$ Martin M Eatock ${ }^{1,10}$ Robert O'Neill, ${ }^{11}$ \\ Fergus Noble, ${ }^{12}$ Timothy J Underwood, ${ }^{13}$ D Paul Harkin, ${ }^{2}$ Manuel Salto-Tellez, \\ Rebecca C Fitzgerald, ${ }^{7}$ Richard D Kennedy, ${ }^{1,2}$ on behalf of the Oesophageal Cancer \\ Clinical and Molecular Stratification (OCCAMS) Study Group
}

\begin{abstract}
- Additional material is published online only. To view please visit the journal online (http://dx.doi.org/10.1136/ gutjnl-2018-317624).
\end{abstract}

For numbered affiliations see end of article.

Correspondence to Dr Richard C Turkington, Centre for Cancer Research and Cell Biology, Queen's University Belfast, Belfast, BT9 7BL, UK; r.turkington@qub.ac.uk

Received 19 September 2018 Revised 13 February 2019 Accepted 15 February 2019 Published Online First 9 March 2019

Check for updates

(C) Author(s) (or their employer(s)) 2019. Re-use permitted under CC BY-NC. No commercial re-use. See rights and permissions. Published by BMJ.

To cite: Turkington $\mathrm{RC}$ Knight LA, Blayney JK, et al. Gut 2019:68:1918-1927.

\section{ABSTRACT}

Objective Current strategies to guide selection of neoadjuvant therapy in oesophageal adenocarcinoma (OAC) are inadequate. We assessed the ability of a DNA damage immune response (DDIR) assay to predict response following neoadjuvant chemotherapy in OAC.

Design Transcriptional profiling of 273 formalin-fixed paraffin-embedded prechemotherapy endoscopic OAC biopsies was performed. All patients were treated with platinum-based neoadjuvant chemotherapy and resection between 2003 and 2014 at four centres in the Oesophageal Cancer Clinical and Molecular Stratification consortium. CD8 and programmed death ligand 1 (PD-L1) immunohistochemical staining was assessed in matched resection specimens from 126 cases. KaplanMeier and Cox proportional hazards regression analysis were applied according to DDIR status for recurrencefree survival (RFS) and overall survival (OS).

Results A total of 66 OAC samples (24\%) were DDIR positive with the remaining 207 samples (76\%) being DDIR negative. DDIR assay positivity was associated with improved RFS (HR: $0.61 ; 95 \% \mathrm{Cl} 0.38$ to 0.98 ; $\mathrm{p}=0.042$ ) and OS (HR: $0.52 ; 95 \% \mathrm{Cl} 0.31$ to 0.88 ; $\mathrm{p}=0.015$ ) following multivariate analysis. DDIR-positive patients had a higher pathological response rate $(p=0.033)$, lower nodal burden $(p=0.026)$ and reduced circumferential margin involvement $(p=0.007)$. No difference in OS was observed according to DDIR status in an independent surgery-alone dataset. DDIR-positive OAC tumours were also associated with the presence of CD8+ lymphocytes (intratumoural: $p<0.001$; stromal: $p=0.026$ ) as well as PD-L1 expression (intratumoural: $p=0.047$; stromal: $p=0.025$ ).

Conclusion The DDIR assay is strongly predictive of benefit from DNA-damaging neoadjuvant chemotherapy followed by surgical resection and is associated with a proinflammatory microenvironment in OAC.

\section{Significance of this study}

What is already known on this subject?

- Neoadjuvant therapy followed by surgical resection cures less than half of patients with resectable oesophageal adenocarcinoma (OAC).

- Response rates to neoadjuvant platinum-based chemotherapy are low at $15 \%$

- Recent molecular landscape studies in OAC have indicated the presence of a DNA damage response impaired subgroup of tumours.

What are the new findings?

- A 44-gene DNA damage immune response (DDIR) assay can successfully be applied to formalin-fixed paraffin-embedded pretreatment endoscopic biopsies with a success rate of $>98 \%$.

- The DDIR assay is predictive of response and survival benefit following DNA-damaging neoadjuvant chemotherapy and surgery.

- DDIR-positive patients have increased pathological response, lower nodal burden and reduced resection margin involvement.

- DDIR positivity is associated with an inflammatory microenvironment characterised by the presence of CD8-positive tumourinfiltrating lymphocytes and high programmed death ligand 1 expression.

\section{INTRODUCTION}

The incidence of oesophageal adenocarcinoma $(\mathrm{OAC})$ in the Western world has risen sixfold in the last 40 years with the highest incidence occurring in the UK. ${ }^{1-3}$ In resectable cases, the addition of neoadjuvant or perioperative therapy provides a modest improvement in overall survival (OS), but only $15 \%$ of patients demonstrate a histopathological response to therapy in the resected tumour. ${ }^{4-7}$ 
Significance of this study

How might it impact on clinical practice in the foreseeable future?

- The ability to select the appropriate neoadjuvant therapy for individual patients with OAC could increase pathological response rates and survival.

- Ineffective therapy could be avoided in patients with OAC unlikely to respond.

- Insights into the molecular biology of the DDIR subgroup will allow novel combinations of conventional therapy with DNA repair inhibitors or immunotherapy to be explored.

Despite improvements in oncological and surgical management, the majority of patients relapse and die of their cancer. ${ }^{4-6}$ Therefore, there is a pressing need to identify biomarkers capable of predicting response in order to select the appropriate neoadjuvant therapy for individual patients.

Imaging and molecular features of OAC have been studied in an attempt to identify predictive biomarkers to neoadjuvant therapy. For example, serial $\left[{ }^{18} \mathrm{~F}\right]-2$-fluoro-2-deoxy-d-glucose $\left({ }^{18} \mathrm{FDG}\right)$ positron emission tomography (FDG-PET) scans can detect changes in tumour metabolism with the aim of predicting pathological response. ${ }^{8-10}$ A 35\% reduction in standard uptake value (SUV) 14 days after baseline has been correlated with a higher rate of tumour regression, R0 resection and improved survival in a prospective study of resectable OAC. ${ }^{9}$ However, $42 \%$ of FDG-PET responders identified by a reduction in SUV did not in fact achieve a pathological response, highlighting the pressing need to identify more accurate molecular predictive biomarkers. Various proposed single gene predictive biomarkers, such as nuclear-factor- $\kappa \beta$, epidermal growth factor receptor, TP53, ERCC1 and thymidylate synthase, have met with limited success as they fail to capture the complex biology of OAC. ${ }^{11-18}$ Recent advances in the molecular understanding of OAC have demonstrated that it is a disease characterised by a high level of mutations and copy number changes giving rise to prominent intratumoural heterogeneity. ${ }^{19-22}$ To encapsulate the biology underpinning response to chemotherapy in OAC, a number of studies have applied gene expression profiling to pretreatment endoscopic biopsies to identify a predictive gene signature. ${ }^{23-25}$ However, these signatures rely on fresh frozen tissue, which is not routinely available, and have been developed in small discovery cohorts without independent validation.

The DNA damage immune response (DDIR) assay, formerly known as the DNA Damage Response Deficiency assay, was previously developed in breast cancer using an unsupervised hierarchical clustering approach. ${ }^{26}$ When tested in an independent breast cancer dataset $(n=203)$, DDIR positivity was associated with an OR for pathological response following neoadjuvant chemotherapy of 3.96 (95\% CI 1.67 to $9.41 ; \mathrm{p}=0.002)$, and in a cohort of 191 patients with node-negative breast cancer, the assay predicted 5 -year disease-free survival following adjuvant chemotherapy with an $\mathrm{HR}$ of 0.37 (95\% CI 0.15 to 0.88 ; $\mathrm{p}=0.025)$. Further validation in 664 chemo-naive patients indicated that the DDIR assay was not prognostic and only predicts outcome in the context of DNA-damaging chemotherapy. Biologically, the DDIR assay indicates constitutive activation of the cyclic GMP-AMP synthase (cGAS)/stimulator of interferon genes (STING) pathway in response to endogenous DNA damage. ${ }^{27}$ Deficiencies in DNA repair and the Fanconi anaemia/ BRCA pathway in particular have been reported to activate this pathway. Importantly, the 44-gene DDIR assay includes well-known immune checkpoint targets, such as programmed death ligand 1 (PD-L1) and indoleamine 2,3-dioxygenase 1, as well as several inflammatory cytokines. Immune activation via the STING pathway results in infiltration of the tumour by $T$ lymphocytes and upregulation of immune checkpoints to create an inflammatory microenvironment associated with chemosensitivity. However, pathological response (tumour regression grade [TRG] 1/2) to DNA-damaging chemotherapy and chemoradiotherapy occurs in only $15 \%$ and $23 \%$ of OAC tumours, respectively. ${ }^{67}$ We hypothesised that pathological tumour response and improved survival may be due to pre-existing deficiencies in DNA repair pathways with associated activation of an innate immune response. An assay that could identify this subgroup of OAC tumours would predict benefit from neoadjuvant chemotherapy.

We, therefore, assessed the ability of the DDIR assay to predict pathological response and prognosis following DNA-damaging neoadjuvant chemotherapy in OAC. We demonstrate that the DDIR assay can be applied to routine diagnostic clinical specimens to allow the selection of patients for whom DNA-damaging chemotherapy would be beneficial. DDIR positivity is also strongly correlated with the presence of tumour-infiltrating lymphocytes (TILs) and PD-L1 expression indicating an association between deficiencies in DNA damage repair mechanisms and a proinflammatory microenvironment in OAC.

\section{MATERIALS AND METHODS}

This study was performed according to the REporting recommendations for tumour MARKer prognostic studies (REMARK) as outlined in the criteria checklist (online supplementary table 1) and REMARK study design diagram (online supplementary figure 1 and online appendix A)

\section{Patient samples}

FFPE prechemotherapy endoscopic biopsies from 273 patients with resectable OAC, treated with neoadjuvant chemotherapy followed by surgical resection, were collected at four UK centres in the Oesophageal Cancer Clinical and Molecular Stratification (OCCAMS) consortium between 2003 and 2014 (online supplementary table 2). Follow-up was performed according to local institutional guidelines. Patients with localised histologically confirmed adenocarcinoma of the oesophagus or gastro-oesophageal junction were included, and all patients were followed up for at least 2 years. Pathological response was assessed in the matched resection specimens according to the method described by Mandard et al with a responder defined as TRG $\leq 2.728$ Assuming a marker positive rate of $21 \%$ (estimated from preliminary data), a sample set of 273 patients had an $80 \%$ power to detect a HR of 2.

For independent in silico validation, a publicly available dataset of 57 OAC resections that did not receive DNA-damaging chemotherapy (GSE19417) was assessed (online supplementary table 3). All tumour samples were collected and snap-frozen from patients undergoing potentially curative surgical resection at the Bristol Royal Infirmary between 1992 and 2000. Gene expression profiling was performed using a custom-made Agilent $44 \mathrm{~K}$ 60 -mer oligomicroarray as previously described. ${ }^{29}$

\section{Gene expression profiling from FFPE tissue}

Biopsies were reviewed for pathological subtype prior to marking for macrodissection and samples containing at least 50\% adenocarcinoma tissue by area were taken forward. Where tumour material was limited endoscopic biopsy fragments from the same 
patient were pooled. Total RNA was extracted using the Recoverall Total Nucleic Acid Isolation Kit for FFPE (Thermo Fisher Scientific, Waltham, Massachusetts, USA) and amplified using the NuGen Ovation FFPE Amplification System v3 (NuGen San Carlos, California, USA). The amplified product was hybridised to the Almac Diagnostics Xcel array (Almac, Craigavon, UK), a cDNA microarray-based technology optimised for archival FFPE tissue and analysed using the Affymetrix Genechip 7G scanner (Affymetrix, Santa Clara, California, USA) as previously described. ${ }^{2630}$ Functional enrichment was performed using the Database for Annotation, Visualization and Integrated Discovery. Raw expression data are available at the Array Express repository (Accession Number E-MTAB-6969).

\section{Immunohistochemistry (IHC)}

Matched FFPE OAC resection specimens were available for 126 patients who received neoadjuvant chemotherapy prior to surgical resection at the Northern Ireland Cancer Centre. Pathological staging was defined according to International Union Against Cancer TNM Staging, 7th Edition, and the cases had a median follow-up time of 48.8 months (online supplementary table 4). All cases were represented in triplicate, and the Tissue Microarray (TMA) was constructed as previously described. ${ }^{31} 32$

Antibodies to CD8 (C8/144B, M7103, Dako) and PD-L1 (SP142, Roche) were used as previously described. ${ }^{27}$ TMA sections were scored by two independent observers (EEP and EM) who were blinded to the clinical data. A semiquantitative scoring system was used for CD8 + expression with a score of 3 indicating strong CD8 + expression, 2 moderate expression, 1 weak expression and 0 absence of expression. For PD-L1, tumour and stroma were scored for percentage of cells with positive expression and previously published cut-offs of $1 \%$ or greater and $5 \%$ or greater were used for analysis. ${ }^{33}$

\section{Whole genome sequencing}

Matched whole genome sequencing data were available for 44 patients who received neoadjuvant chemotherapy prior to surgical resection at three OCCAMS centres (Cambridge, Edinburgh and Southampton; online supplementary table 4).

Whole genome sequencing was performed, and mutational signatures were identified using the non-negative matrix factorisation (NMF) methodology as previously described. ${ }^{22} 34$

\section{Statistical analysis}

Microarray data were preprocessed using the Robust Multiarray Average model for the Almac Diagnostics Xcel array with DDIR signature scores calculated and predefined cut-points applied as previously described. ${ }^{26}$ A threshold of 0.3403 was optimised in an independent technical study of $n=45$ OAC samples and applied independently to the validation cohort dichotomising patients as DDIR positive $(>0.3403)$ or DDIR negative $(\leq 0.3403)$. Cox proportional hazards regression was used to investigate the prognostic effects of the DDIR signature on relapse-free survival (RFS) and OS defined as the time from surgical resection to relapse of disease or death from any cause, respectively. The estimated effect of the signature was adjusted for factors available at the time of diagnosis (clinical tumour status, clinical nodal status and tumour grade) by fitting a multivariate model.

Further details are available in the online supplementary methods.
RESULTS

Assessment of the DDIR assay and survival following neoadjuvant chemotherapy and surgical resection in OAC

To assess the ability of the DDIR assay to predict survival following neoadjuvant DNA-damaging chemotherapy and resection in OAC, it was applied to a retrospective dataset of 273 FFPE biopsy samples. A total of 66 OAC samples (24\%) were characterised as DDIR positive with the remaining 207 (76\%) being DDIR negative. Reductions in lymph node and circumferential resection margin (CRM) involvement, a more proximal tumour location and older age were observed for DDIR positive tumours (table 1). DDIR assay positivity was associated with improved RFS (HR $0.58,95 \%$ CI 0.38 to $0.90 ; \mathrm{p}=0.015$ ) and OS (HR $0.62,95 \%$ CI 0.41 to $0.95 ; \mathrm{p}=0.029$ ) following surgical resection (figure 1). When evaluated as a continuous variable, higher DDIR scores were associated with both improved RFS (HR 0.34, 95\% CI 0.13 to $0.93 ; \mathrm{p}=0.036$ ) and OS (HR 0.32, $95 \%$ CI 0.12 to $0.87 ; \mathrm{p}=0.026$ ). Univariate analysis confirmed associations between survival and presurgical clinical $\mathrm{N}$ stage as well as postsurgical factors such as pathological $\mathrm{T}$ and $\mathrm{N}$ stage, differentiation, lymphovascular invasion and CRM status (online supplementary table 5). Applying a published cut-off of $\geq 15$ lymph nodes to indicate an adequate lymph node yield, we found that there was no association between the DDIR status and lymph node yield and neither was there an association between adequate lymph node yield and RFS (HR 0.94, 95\% CI 0.66 to $1.39 ; \mathrm{P}=0.847$ ) or OS (HR $1.02,95 \% \mathrm{CI} 0.7$ to 1.48 ; $\mathrm{p}=0.916$ ). While the Lauren classification is known to be prognostic in OAC, it was not available for the whole cohort and so it is unclear how the DDIR assay relates to intestinal versus diffuse type adenocarcinomas. ${ }^{35}$ Also, there was no association between the administration of postoperative chemotherapy and DDIR status (HR $0.74,95 \%$ CI 0.39 to $1.4 ; \mathrm{p}=0.354$ ), but there was a trend towards improved OS in DDIR-positive patients when no adjuvant chemotherapy was administered (HR 0.39, 95\% CI 0.15 to $1.02 ; \mathrm{p}=0.55$ ) (online supplementary figure 2 ).

Multivariable analysis was performed to test the association between DDIR status and each survival endpoint following adjustment for factors available at diagnosis (table 2). DDIR-positive patients had improved RFS relative to DDIR negative patients (HR $0.61,95 \%$ CI 0.38 to $0.98 ; \mathrm{p}=0.042$ ), and assay positivity was also independently associated with improved OS (HR 0.52 , 95\% CI 0.31 to $0.88 ; \mathrm{p}=0.015$ ).

To assess whether the DDIR assay was prognostic, independent of DNA-damaging chemotherapy treatment, it was applied to a publicly available dataset of 57 OAC resections, which did not receive neoadjuvant chemotherapy (online supplementary table 3). No significant difference in overall survival was noted between the DDIR-positive and DDIR-negative populations (HR $0.86,95 \%$ CI 0.48 to 1.55 ; p=0.61) (online supplementary figure 3 ). However, further confirmatory results in a larger cohort are required. Taken together, these results indicate that the DDIR assay is a strong predictor of survival benefit following surgical resection in OAC, but only in the context of neoadjuvant DNA-damaging chemotherapy.

\section{The DDIR assay is predictive of pathological response in OAC}

The ability to predict pathological response to neoadjuvant chemotherapy would improve patient stratification and treatment selection in OAC. TRG was available for 228 patients in the OAC cohort with $24(11 \%)$ of cases having a TRG $\leq 2$, indicating a pathological response, and 203 (89\%) TRG 3-5, in keeping with limited or no response to chemotherapy. Pathological 
Table 1 Association of clinicopathological characteristics with DDIR status in the OAC cohort

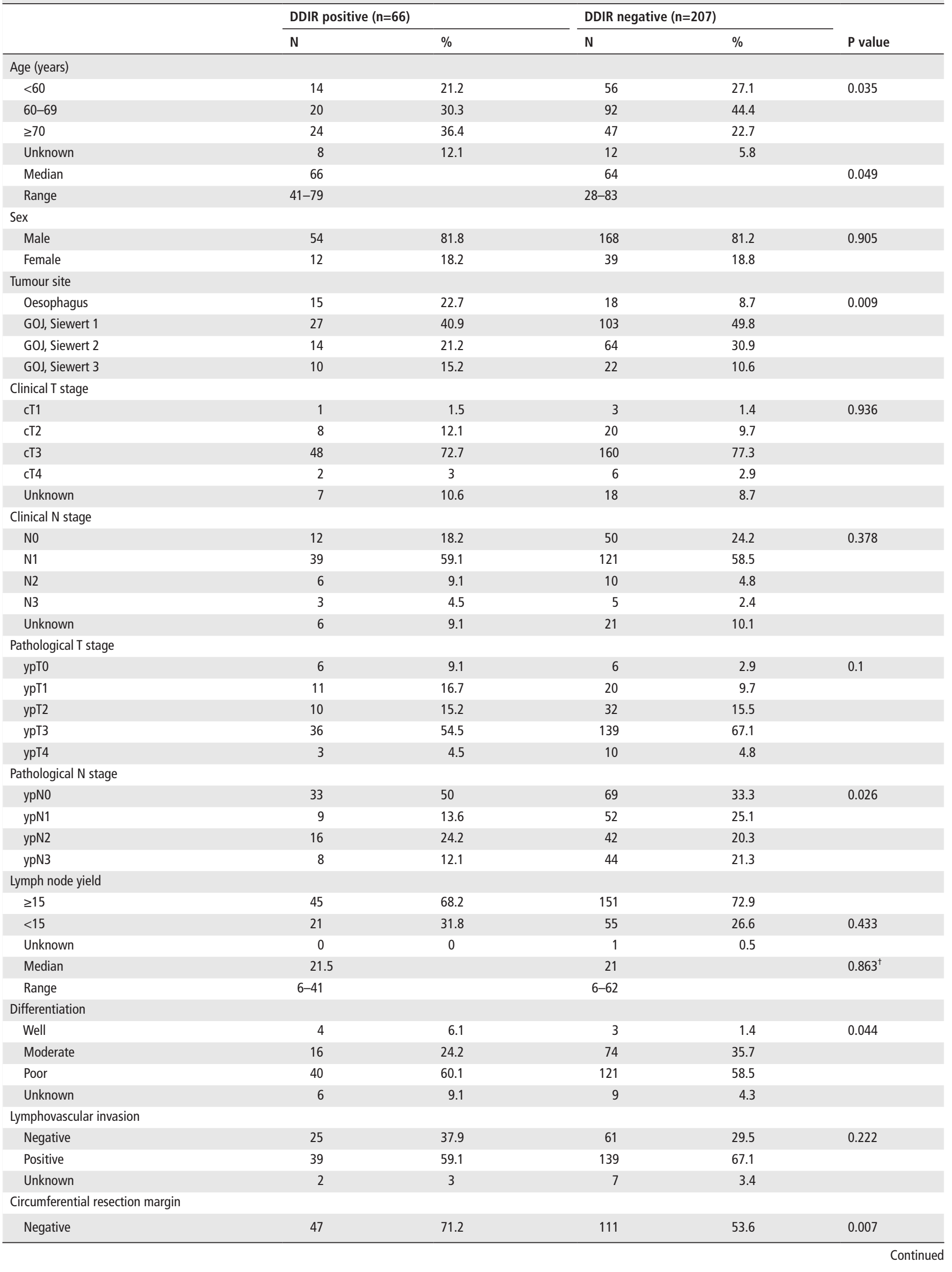




\begin{tabular}{|c|c|c|c|c|c|}
\hline & \multicolumn{2}{|c|}{ DDIR positive $(n=66)$} & \multicolumn{2}{|c|}{ DDIR negative $(n=207)$} & \multirow[b]{2}{*}{$P$ value } \\
\hline & $\mathrm{N}$ & $\%$ & $\mathrm{~N}$ & $\%$ & \\
\hline Positive & 15 & 22.7 & 85 & 41.1 & \\
\hline Unknown & 4 & 6.1 & 11 & 5.3 & \\
\hline \multicolumn{6}{|c|}{ Neoadjuvant chemotherapy } \\
\hline CFU/CX & 12 & 18.2 & 33 & 15.9 & 0.89 \\
\hline ECF/X & 52 & 78.8 & 168 & 81.2 & \\
\hline Oxaliplatin/X & 1 & 1.5 & 4 & 1.9 & \\
\hline Unknown & 1 & 1.5 & 2 & 1 & \\
\hline \multicolumn{6}{|c|}{ Adjuvant chemotherapy received } \\
\hline No & 12 & 18.2 & 48 & 23.2 & 0.448 \\
\hline Yes & 26 & 39.4 & 75 & 36.2 & \\
\hline Unknown & 28 & 42.4 & 84 & 40.6 & \\
\hline \multicolumn{6}{|c|}{ Pathological response } \\
\hline Responder & 11 & 16.7 & 14 & 6.8 & 0.025 \\
\hline Non-responder & 45 & 68.2 & 158 & 76.3 & \\
\hline Unknown & 10 & 15.2 & 35 & 16.9 & \\
\hline
\end{tabular}

Mann-Whitney U test.

CFU, cisplatin and 5-fluorouracil; CX, cisplatin and capecitabine (xeloda); DDIR, DNA damage immune response; ECF/X, epirubicin, cisplatin and 5-fluorouracil/capecitabine (xeloda); GOJ, gastro-oesophageal junction; OAC, oesophageal adenocarcinoma; Oxaliplatin/X, oxaliplatin and capecitabine (xeloda).

response was observed in $16.7 \%$ and $6.8 \%$ of DDIR-positive and DDIR-negative cases, respectively $(p=0.025)$ (table 1$)$. DDIR scores were grouped by response status and one-way analysis of variance analysis demonstrated significantly higher DDIR scores in responders compared with non-responders $(p=0.033)$. This indicates that the DDIR score was significantly enriched for tumours that respond to neoadjuvant chemotherapy in OAC (figure 2).

\section{DDIR assay positivity and tumour mutational load}

Recent sequencing studies have stratified OAC into subtypes defined by the pattern of somatic mutations. Secrier $e t a l^{22}$ identified three subgroups $(\mathrm{C}>\mathrm{A} / \mathrm{T}$ dominant, DNA damage response [DDR] impaired and mutagenic through the application of mutational signatures to WGS data from a cohort of 129 chemotherapy-naïve OAC samples. We sought to assess the overlap between cases defined as DDIR positive by our gene expression assay and DDR impaired by mutational signature analysis. A total of 44 cases had matched gene expression and WGS data available and demonstrated higher clinical nodal staging and different neoadjuvant chemotherapy regimens compared with the whole cohort and the tissue microarray (TMA) subset (online supplementary table 4). This may reflect differing staging methodologies used at the largest contributing centre to the WGS cohort (University of Cambridge; 29 [66\%] of patients) and the increased use of cisplatin and oxaliplatin doublet neoadjuvant regimens due to clinical trials recruiting at that centre at the time of sample collection $\left(\mathrm{OEO}^{36}\right.$ and $\left.\mathrm{LEO}^{37}\right)$.

NMF was applied to cluster the patients into the three subgroups (online supplementary figure 4). No association was observed between the DDIR status and the predominant mutational signature (online supplementary table $6 ; \mathrm{p}=0.83$ ). Although the size of the cohort limits the statistical power of the analysis, DDIR-positive patients did display a trend towards higher tumour mutational burden and a higher mutation rate (online supplementary figure 5). However, no significant differences were observed in the mean copy number or total number of deleterious somatic mutations or indels in multiple DDR pathways between the DDIR-positive and DDIR-negative samples.
Neither were there any differences observed in the copy number of genes involved in the homologous recombination, double and single strand break repair pathways (data not shown). While both assays are related to loss of DNA repair, the assessment of differing biologies represented by immune activation in response to DNA damage measured by the DDIR assay, as opposed to the pattern of mutations caused by deficiencies in DNA repair mechanisms may lead to the lack of association.

\section{DDIR assay positivity is associated with CD8+ Tlymphocytes and expression of PD-L1}

We hypothesised that increased DNA damage in DDIR-positive tumours may be associated with increased lymphocytic infiltration and upregulation of immune checkpoint genes. A list of 45 genes differentially expressed between DDIR-positive and DDIR-negative patients, with a fold change of $>2$, was generated (online supplementary table 7). As expected, this list included the genes from the DDIR signature, with 5 out of 44 genes represented, but it also included a number of genes encoding inflammatory cytokines and mediators of an immune response. Chemokines such as CXCL9 and CXCL13 showed 5.5-fold and 4.58-fold upregulation, respectively, and 29 of the 45 genes (64.4\%) have a role in the immune response. Pathway analysis demonstrated enrichment of a wide range of biological processes related to immune activation and viral response (online supplementary table 8), further strengthening the association of DDIR-positive status with a proinflammatory microenvironment.

To assess the relationship between DDIR status, PD-L1 expression and the presence of TILs, we performed IHC analysis on 126 resection specimens matched to patients in the gene expression cohort (figure 3, table 3, online supplementary tables 9 and 10$)$. Previously, published cut-offs of $1 \%$ or greater and $5 \%$ or greater were used to define PD-L1 positivity. A statistically significant association was observed between DDIR assay positivity and intratumoural and stromal PD-L1 expression at the $5 \%$ cut-off $(\mathrm{p}=0.047 ; \mathrm{p}=0.25$, respectively). The presence of both intratumoural and stromal CD8 + TILs was also associated with DDIR assay positivity $(p<0.001 ; p=0.026$, respectively). 

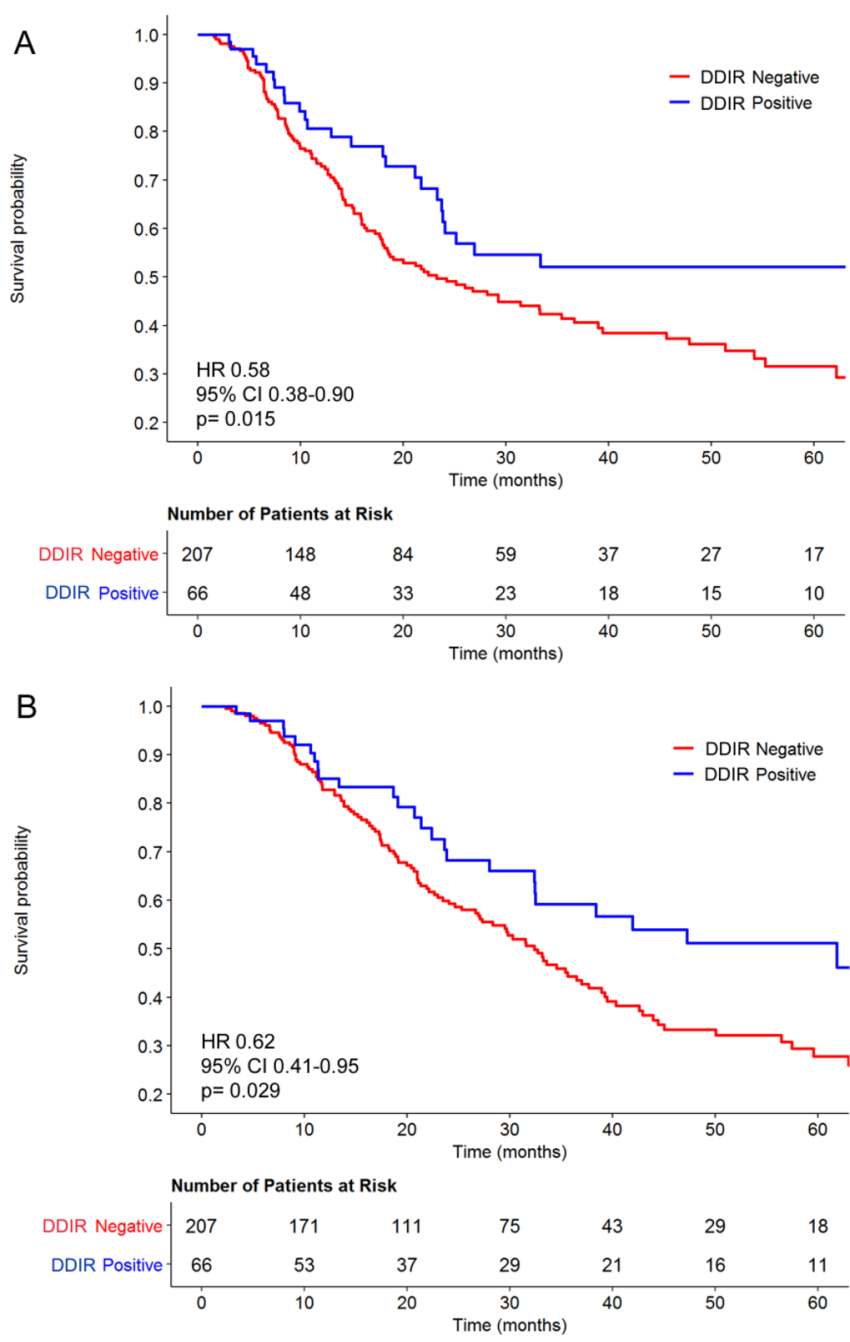

Figure 1 Kaplan-Meier curves stratified by the DDIR assay for (A) relapse-free and (B) overall survival for 273 patients with oesophageal adenocarcinoma treated with cisplatin-based neoadjuvant chemotherapy followed by surgical resection. DDIR, DNA damage immune response.

\section{DISCUSSION}

We have demonstrated that the DDIR assay is predictive of response and independently prognostic following DNA-damaging neoadjuvant chemotherapy and surgical resection in OAC. DDIR assay positivity was associated with improved survival following chemotherapy and surgery and identified those patients with a higher probability of obtaining a pathological

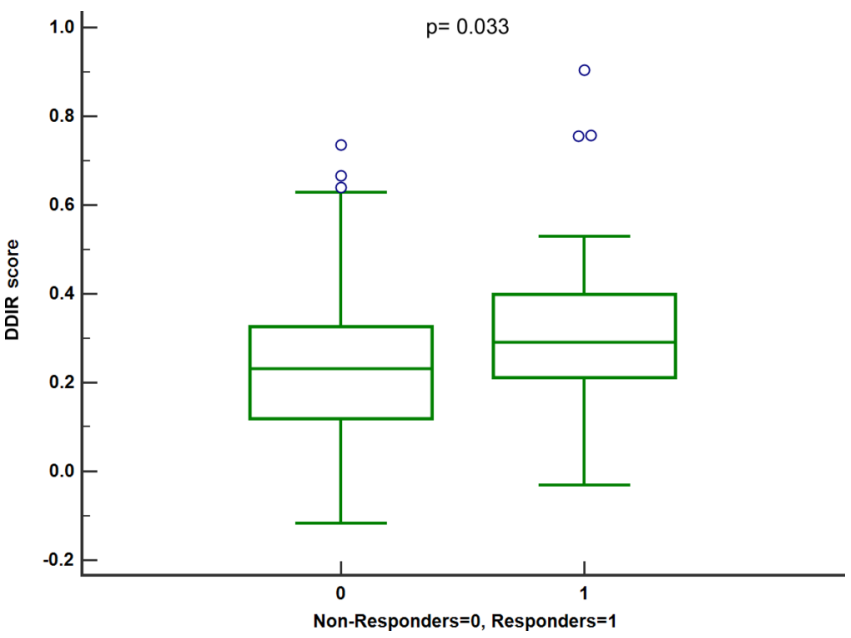

Figure 2 Boxplot of DDIR scores grouped by response status. DDIR, DNA damage immune response.

response, reduced nodal burden and clear resection margins. When assessed alongside clinical factors available at the time of diagnosis, DDIR status demonstrated superior prognostic ability compared with standard clinicopathological factors. Application of the DDIR assay to a cohort of patients who did not receive neoadjuvant therapy demonstrated no difference in survival according to DDIR status indicating that the DDIR assay may not be prognostic in its own right but only in the context of DNA-damaging therapy.

Our study has a number of advantages compared with prior attempts to identify a predictive biomarker to neoadjuvant therapy in OAC. Previous biomarker studies have relied on fresh frozen tissue, which is not routinely collected, and suffered from high attrition rates for samples analysis. However, our study used FFPE diagnostic tissue with a success rate of $95.8 \%$ in samples submitted for analysis, allowing the assay to be readily applied to clinical practice. Other attempts to develop a predictive classifier have also been limited by small sample size and lack of suitable validation sets. ${ }^{23-2538}$ We were able to validate the DDIR assay in a sufficiently powered real-world cohort of patients to assess its predictive ability, and the assay has also undergone extensive analytical validation enabling it to be reproducibly applied to clinical samples.

Limitations of the study include the use of a retrospective clinical cohort that may influence survival outcomes due to the absence of standardised follow-up procedures and so the DDIR assay will require further validation in a randomised controlled trial dataset and by a prospective study. Also, all patients were

Table 2 Multivariate analysis and combined model of clinicopathological factors, DDIR status, relapse-free and overall survival in OAC

\begin{tabular}{|c|c|c|c|c|c|c|}
\hline & \multicolumn{3}{|c|}{ Relapse-free survival } & \multicolumn{3}{|c|}{ Overall survival } \\
\hline & $\mathrm{HR}$ & $95 \% \mathrm{Cl}$ & $P$ value & HR & $95 \% \mathrm{Cl}$ & $P$ value \\
\hline \multicolumn{7}{|l|}{ Multivariate model } \\
\hline $\begin{array}{l}\text { Clinical T stage } \\
\text { (T1/2 v } 3 / 4)\end{array}$ & 1.08 & $0.56-2.09$ & 0.810 & 1.05 & $0.55-2.03$ & 0.876 \\
\hline $\begin{array}{l}\text { Clinical N stage } \\
\text { (N0 v 1/2/3) }\end{array}$ & 1.67 & $1.04-2.67$ & 0.033 & 1.51 & $1.94-2.42$ & 0.088 \\
\hline
\end{tabular}

DDIR, DNA damage immune response; OAC, oesophageal adenocarcinoma. 


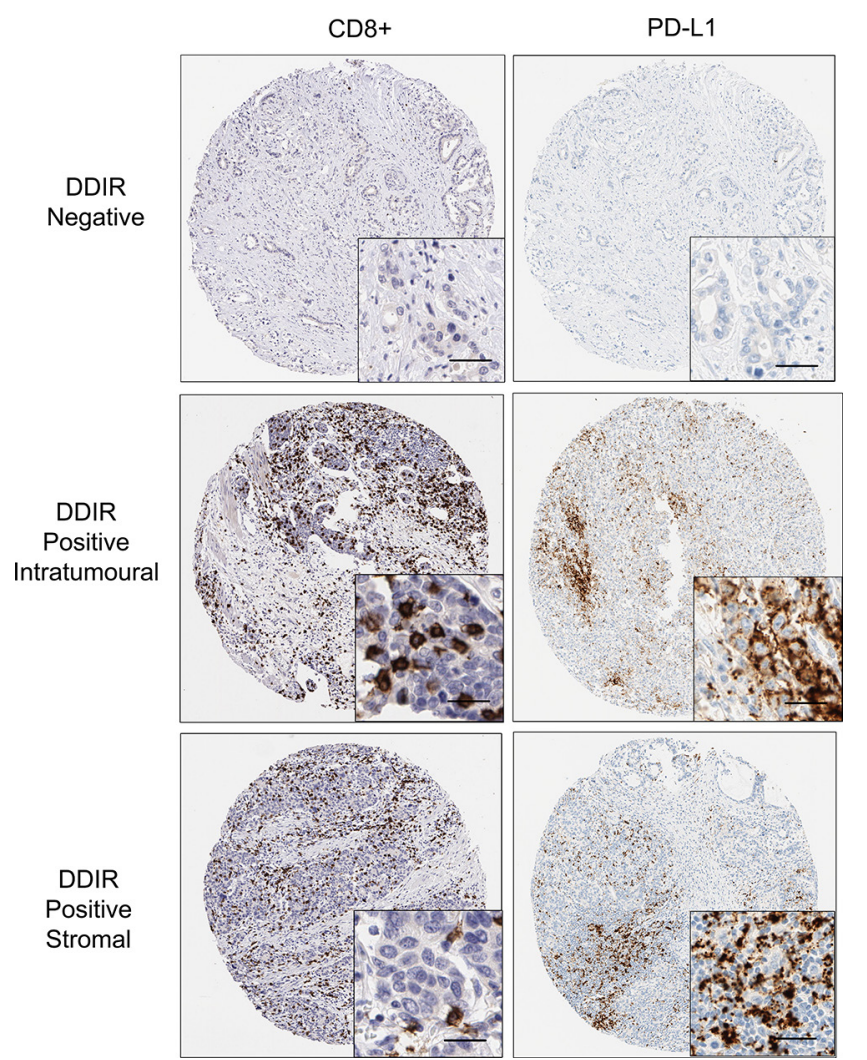

Figure 3 Immunohistochemistry images $(\times 10$; inset $\times 40)$ showing absence of CD8+ lymphocytes and PD-L1 staining in DDIR assaynegative tumours. Both intratumoural and stromal CD8+ lymphocytes were observed in DDIR assay-positive tumours along with PD-L1 tumours. Scale bar represents $50 \mu \mathrm{M}$. DDIR, DNA damage immune response; PD-L1, programmed death ligand 1.

treated with neoadjuvant platinum-based chemotherapy prior to surgical resection. Considering neoadjuvant chemoradiotherapy is standard practice in the USA and many parts of Europe; further validation is required in a sample set treated with this modality. An additional challenge for many biomarker studies is the heterogeneity demonstrated by OAC. A high level of intratumoural heterogeneity has been correlated with response to neoadjuvant chemotherapy in OAC and has indicated the limitations of a single biopsy to develop a predictive biomarker. ${ }^{39}$ This may be partially mitigated in our study by the pooling of endoscopic biopsy fragments with sufficient tumour material, but only samples from multiple sites within the tumour could encompass the underlying clonality of OAC tumours. The limited amount of tumour tissue available in the biopsy samples also precluded their use in the analysis of TILs and PD-L1 expression and so matched resection specimens were used. However, the prior administration of neoadjuvant chemotherapy may have influenced the amount of TILs present and the expression levels of PD-L1 in these specimens.

With regard to the clinical applicability of the assay, a number of factors should be taken into consideration. The response rate of $16.7 \%$ observed in DDIR-positive patients was significantly higher than that observed in DDIR-negative patients $(6.8 \%)$, but is comparable with unselected published retrospective and clinical trial cohorts. ${ }^{4}$ This may limit the utility of the assay as a tool to enhance pathological response following neoadjuvant chemotherapy. Conversely, a response rate of $6.8 \%$ in DDIR-negative patients may not be low enough to dissuade
Table 3 CD8+ intratumoural and stromal lymphocytic infiltrate and PD-L1 staining assessed by IHC in DDIR-positive and DDIR-negative tumours

\begin{tabular}{|c|c|c|c|c|c|}
\hline & \multicolumn{2}{|c|}{$\begin{array}{l}\text { DDIR positive } \\
(n=24)\end{array}$} & \multicolumn{2}{|c|}{$\begin{array}{l}\text { DDIR negative } \\
(n=102)\end{array}$} & \multirow[b]{2}{*}{$P$ value } \\
\hline & $\mathrm{N}$ & $\%$ & $\mathrm{~N}$ & $\%$ & \\
\hline \multicolumn{6}{|c|}{ Intratumoural } \\
\hline \multicolumn{6}{|l|}{ PD-L1 } \\
\hline$\geq 1 \%$ & 7 & 29.2 & 10 & 9.8 & 0.02 \\
\hline$<1 \%$ & 17 & 70.8 & 92 & 90.2 & \\
\hline$\geq 5 \%$ & 3 & 12.5 & 2 & 2 & 0.047 \\
\hline$<5 \%$ & 21 & 70.8 & 100 & 90.2 & \\
\hline \multicolumn{6}{|l|}{ CD8+ } \\
\hline 3 & 1 & 4.2 & 0 & 0 & $<0.001$ \\
\hline 2 & 4 & 16.7 & 1 & 1 & \\
\hline 1 & 14 & 13.7 & 63 & 61.7 & \\
\hline 0 & 5 & 4.9 & 38 & 37.2 & \\
\hline \multicolumn{6}{|l|}{ Stromal } \\
\hline \multicolumn{6}{|l|}{ PD-L1 } \\
\hline$\geq 1 \%$ & 17 & 70.8 & 52 & 51 & 0.11 \\
\hline$<1 \%$ & 7 & 29.2 & 50 & 49 & \\
\hline$\geq 5 \%$ & 8 & 33.3 & 12 & 11.8 & 0.025 \\
\hline$<5 \%$ & 16 & 66.7 & 90 & 88.2 & \\
\hline \multicolumn{6}{|l|}{ CD8+ } \\
\hline 3 & 8 & 33.3 & 10 & 9.8 & 0.026 \\
\hline 2 & 8 & 33.3 & 45 & 44.1 & \\
\hline 1 & 8 & 33.3 & 44 & 43.1 & \\
\hline 0 & 0 & 0 & 3 & 2.9 & \\
\hline
\end{tabular}

DDIR, DNA damage immune response; PD-L1, programmed death ligand 1.

clinicians from using neoadjuvant cisplatin-based chemotherapy in this patient population. Data from other cancer types indicating an increase in response following taxane treatment in tumours with intact DNA repair mechanisms may provide a rationale for the use of the docetaxel, oxaliplatin, fluorouracil/ leucovorin chemotherapy (FLOT) regimen in DDIR-negative patients. For example, ovarian cancer patients with low/intermediate levels of BRCA1 have improved survival following treatment with platinum-based chemotherapy, whereas high levels of BRCA1 expression correlate with improved OS following the use of taxane-contatining chemotherapy. ${ }^{40}$ Similarly, in breast cancer cell lines, exogenous expression of BRCA1 increased sensitivity to spindle poisons, such as paclitaxel and vinorelbine. ${ }^{41}$ We would hypothesise that DDIR-positive patients may benefit from the direct damage to DNA induced by cisplatin or radiotherapy, whereas the DDIR-negative cases may also require the addition of inhibitors of microtubule formation, such as docetaxel. Testing of the assay in sufficiently powered randomised trial cohorts containing suitable treatment regimens could answer such a question. Further considerations regarding the utility of the assay include the association of DDIR positivity with older patients that could indicate an increased prevalence of this proinflammatory subgroup with increasing age. Also, the trend towards increased survival for DDIR-positive patients who do not receive adjuvant chemotherapy should be interpreted with caution as it is likely to be confounded by patients who had an excellent pathological response not going on to receive further chemotherapy.

The biology of a DNA repair-deficient subgroup should be examined in the context of recent publications from collaborative sequencing efforts that have characterised the molecular 
landscape of oesophagogastric adenocarcinoma. ${ }^{19204243}$ Multiple platform analysis by The Cancer Genome Atlas has identified four subgroups within oesophagogastric adenocarcinoma with tumours of the distal oesophagus and gastro-oesophageal junction characterised by chromosomal instability, a paucity of oncogenic driver mutations and frequent amplifications of upstream activators of signalling pathways. ${ }^{19}{ }^{42} 43$ Within the stomach, tumours may also be of the genomically stable or mismatch repair subtype with the final subgroup of Epstein-Barr virus (EBV)-positive tumours occurring in the distal stomach. ${ }^{42} 43$ Mutational signature analysis of whole genome sequencing data from 129 chemotherapy-naïve OAC samples has revealed three subgroups demonstrating either deficiencies in DNA damage repair, high mutational burden or a $\mathrm{C}>\mathrm{A} / \mathrm{T}$ mutational pattern. ${ }^{22}$ The DDR-impaired subgroup constitutes $20 \%$ of patients with OAC and, while this is in keeping with a DDIR positive rate of $24 \%$, our analysis has shown no significant overlap between the two subgroups. Reasons for this discrepancy could include the differing methodologies used to define DNA repair defects between the sequencing and gene expression dataset, the lack of a defined cut-point to call DDR impaired status in the WGS data and the limited sample size. Also, the DDIR assay takes a functional approach, capturing the inflammatory response activated by DNA damage, whereas analysis of the sequencing data assesses the pattern of mutations that occur as a result of loss of DNA repair. Furthermore, Janjigian et al performed prospective sequencing of 295 patients with metastatic oesophagogastric cancer using a capture-based next-generation sequencing (NGS) platform capable of detecting mutations, copy number alterations and selected rearrangements in up to 468 cancer genes. No single mutant allele or gene with a role in DNA repair was associated with immune response, and a surrogate marker of homologous recombination deficiency, termed the large scale transition (LST) score, was not associated with improved progression-free survival (HR 0.99, $\mathrm{p}=0.947$ ) following firstline platinum-based chemotherapy. Higher LST scores were not observed in patients with response to first-line therapy lasting over 24 months $(p=0.6)$ and neither did the majority of patients with prolonged responses harbour somatic alterations in know HR genes. Conversely, Smyth et al showed that assessment of homologous recombination deficiency using a genomic signature for loss of heterozygosity (LOH) derived from an NGS panel could identify a high LOH group of patients with prolonged survival following platinum-based chemotherapy. ${ }^{44}$ However, this study was limited by a high attrition rate for $\mathrm{LOH}$ inference ( $47 \%$ of samples successfully scored) and small sample numbers. A possible explanation for these results is the limitations imposed by targeted platforms that are unable to detect alterations in genes absent from the panel as well as epigenetic and transcriptional consequences of somatic mutations. Previous studies in breast cancer have shown that while BRCA1/2 mutations may confer sensitivity to DNA-damaging chemotherapy, this is not true for all cases as not all mutations may affect DNA repair or may be compensated for by alternate mechanisms. ${ }^{45}$ Conversely, BRCA1/2 wild-type tumours can possess an abnormal DNA damage response due to epigenetic silencing of BRCA1/2. ${ }^{46} 47$ Therefore, it is likely that the transcriptome-based DDIR assay is capable of capturing the downstream effects of genomic and epigenetic changes and so detect a broader range of mechanisms of DDR impairment. It is clear that a subgroup of patients with DNA repair deficiencies exists within OAC, and further work is needed to accurately characterise this patient group.

Recently, the field of DNA repair biology has enjoyed renewed interest due to its involvement in the immune response to cancer.
Increased DNA damage within cancer cells has been shown to generate a highly immunogenic state within the tumours leading to the presence of TILs and the upregulation of suppressors of the immune response, such as PD-L1 ${ }^{48}$ Our data indicate a strong association between DDIR positivity and an immunogenic microenvironment. Indeed, our group has demonstrated the role of the cGAS-STING pathway in the response to DNA damage with the resultant upregulation of inflammatory cytokines such as CXCL10 and CCL5 as well as PD-L1. ${ }^{27}$ The STING pathway is activated by cytosolic DNA released from the nucleus in response to DNA damage, driving an innate immune type 1 interferon response and a subsequent upregulation of immune checkpoints including PD-L1, a key component of the DDIR signature. Furthermore, the cGAS-STING pathway has been shown to be a key player in response to immune checkpoint blockade. ${ }^{49} 50$ In keeping with this, we demonstrated increased CD8 + Tcell infiltration and PD-L1 expression in DDIR-positive oesophageal tumours, both of which have been proposed as predictive biomarkers for immunotherapy agents. ${ }^{51}{ }^{52}$ The presence of a DNA damage-deficient subgroup in oesophagogastric cancer may indicate sensitivity to conventional chemotherapy and response to immune checkpoint targeted agents.

In summary we have developed an array-based classifier using pretreatment FFPE biopsies to predict benefit from, and response to, neoadjuvant therapy in resectable OAC. The assay is readily applicable to routine pathological samples with potential for rapid translation into clinical use. The identification of a subgroup of tumours with deficiencies in their DNA repair mechanisms will enable these patients to be selected for more effective therapy and improve survival outcomes. Also, knowing the underlying biology of these tumours allows the possibility of further enhancing response to therapy through combinations with novel inhibitors of DNA repair and immunotherapy. Overall, the DDIR assay enables treatment selection and patient stratification in oesophagogastric adenocarcinoma and may improve response to therapy, resection rates and survival in this poor prognostic disease.

\section{Author affiliations}

${ }^{1}$ Centre for Cancer Research and Cell Biology, Queen's University Belfast, Belfast, UK ${ }^{2}$ Almac Diagnostics Ltd, Craigavon, UK

${ }^{3}$ Genetics Institute, University College London, London, UK

${ }^{4}$ Department of Pathology, Belfast Health and Social Care Trust, Belfast, UK

${ }^{5}$ Cancer Research UK Cambridge Institute, University of Cambridge, Cambridge, UK

${ }^{6}$ Translational Gastroenterology Unit, John Radcliffe Hospital Oxford University

Hospitals NHS Trust, Oxford, UK

${ }^{7}$ Hutchison/MRC Research Centre, Cambridge, UK

${ }^{8}$ Department of Histopathology, Addenbrookes Hospital, Cambridge, UK

${ }^{9}$ Northern Ireland Molecular Pathology Laboratory, Queen's University Belfast,

Belfast, UK

${ }^{10}$ Department of Medical Oncology, Belfast Health and Social Care Trust, Belfast, UK

${ }^{11}$ Edinburgh Cancer Research Centre, University of Edinburgh, Edinburgh, UK

${ }^{12}$ Department of Surgery, University Hospital Southampton NHS Foundation Trust,

Southampton, UK

${ }^{13}$ Cancer Sciences Division, University of Southampton, Southampton, UK

Correction notice This article has been corrected since it published Online First. The author correspondence details have been corrected.

Acknowledgements This work was supported by the Gastrointestinal Cancer Research Charitable Fund administered by the Belfast Health and Social Care Trust, the Cancer Research UK Experimental Cancer Medicine Centre Initiative, Invest Northern Ireland and Almac Diagnostics. Oesophageal Cancer Clinical and Molecular Stratification (OCCAMS) was funded by a programme grant from Cancer Research UK (RG66287). We would like to thank the Human Research Tissue Bank, which is supported by the National Institute for Health Research (NIHR) Cambridge Biomedical Research Centre from Addenbrooke's Hospital. Additional infrastructure support was provided from the CRUK funded Experimental Cancer Medicine Centre. RF has programmatic funding from the Medical Research Council and infrastructure support from the NIHR Biomedical Research Centre and the 
Cambridge Experimental Medicine Centre. Tissue samples used in this research were received from the Northern Ireland Biobank, which is funded by HSC Research and Development Division of the Public Health Agency in Northern Ireland and Cancer Research UK through the Belfast Cancer Research UK Centre and the Northern Ireland Experimental Cancer Medicine Centre; additional support was received from the Friends of the Cancer Centre. The Northern Ireland Molecular Pathology Laboratory has received funding from Cancer Research UK, the Friends of the Cancer Centre and the Sean Crummey Foundation. This project has received funding from the European Union's Horizon 2020 research and innovation programme under the Marie Sklodowska-Curie grant agreement no 721906. The OCCAMS Study Group is a multicentre UK collaboration.

Collaborators On behalf of the OCCAMS Study Group: Ayesha Noorani (Medical Research Council Cancer Unit, Hutchison/Medical Research Council Research Centre, University of Cambridge, Cambridge, UK); Paul A W Edwards (Medical Research Council Cancer Unit, Hutchison/Medical Research Council Research Centre, University of Cambridge, Cambridge, UK, Cancer Research UK Cambridge Institute, University of Cambridge, Cambridge, UK); Nicola Grehan (Medical Research Council Cancer Unit, Hutchison/Medical Research Council Research Centre, University of Cambridge, Cambridge, UK); Barbara Nutzinger (Medical Research Council Cancer Unit, Hutchison/Medical Research Council Research Centre, University of Cambridge, Cambridge, UK); Caitriona Hughes (Medical Research Council Cancer Unit, Hutchison/Medical Research Council Research Centre, University of Cambridge, Cambridge, UK); Elwira Fidziukiewicz (Medical Research Council Cancer Unit, Hutchison/Medical Research Council Research Centre, University of Cambridge, Cambridge, UK); Jason Crawte (Medical Research Council Cancer Unit, Hutchison/ Medical Research Council Research Centre, University of Cambridge, Cambridge, UK); Alex Northrop (Medical Research Council Cancer Unit, Hutchison/Medical Research Council Research Centre, University of Cambridge, Cambridge, UK); Gianmarco Contino (Medical Research Council Cancer Unit, Hutchison/Medical Research Council Research Centre, University of Cambridge, Cambridge, UK); Xiaodun Li (Medical Research Council Cancer Unit, Hutchison/Medical Research Council Research Centre, University of Cambridge, Cambridge, UK); Rachel de la Rue (Medical Research Council Cancer Unit, Hutchison/Medical Research Council Research Centre, University of Cambridge, Cambridge, UK); Maria O'Donovan (Medical Research Council Cancer Unit, Hutchison/Medical Research Council Research Centre, University of Cambridge, Cambridge, UK, Department of Histopathology, Addenbrooke's Hospital, Cambridge, UK) Shalini Malhotra (Medical Research Council Cancer Unit, Hutchison/Medical Research Council Research Centre, University of Cambridge, Cambridge, UK, Department of Histopathology, Addenbrooke's Hospital, Cambridge, UK); Monika Tripathi (Medical Research Council Cancer Unit, Hutchison/ Medical Research Council Research Centre, University of Cambridge, Cambridge, UK, Department of Histopathology, Addenbrooke's Hospital, Cambridge, UK); Simon Tavaré (Cancer Research UK Cambridge Institute, University of Cambridge, Cambridge, UK); Andy G Lynch (Cancer Research UK Cambridge Institute, University of Cambridge, Cambridge, UK); Matthew Eldridge (Cancer Research UK Cambridge Institute, University of Cambridge, Cambridge, UK); Lawrence Bower (Cancer Research UK Cambridge Institute, University of Cambridge, Cambridge, UK); Ginny Devonshire (Cancer Research UK Cambridge Institute, University of Cambridge, Cambridge, UK); Sriganesh Jammula (Cancer Research UK Cambridge Institute, University of Cambridge, Cambridge, UK); Jim Davies (Department of Computer Science, University of Oxford, Oxford, UK); Charles Crichton (Department of Computer Science, University of Oxford, Oxford, UK); Nick Carroll (Cambridge University Hospitals NHS Foundation Trust, Cambridge, Cambridge, UK); Peter Safranek (Cambridge University Hospitals NHS Foundation Trust, Cambridge, Cambridge, UK); Andrew Hindmarsh (Cambridge University Hospitals NHS Foundation Trust, Cambridge, UK); Vijayendran Sujendran (Cambridge University Hospitals NHS Foundation Trust, Cambridge, UK); Stephen J Hayes (Salford Royal NHS Foundation Trust, Salford, UK; Faculty of Medicaland Human Sciences, University of Manchester, Manchester, UK); Yeng Ang (Salford Royal NHS Foundation Trust, Salford, UK; Wigan and Leigh NHS Foundation Trust, Wigan, Manchester, UK; GI Science Centre, University of Manchester, Manchester, UK); Shaun R Preston (Royal Surrey County Hospital NHS Foundation Trust, Guildford, UK); Sarah Oakes (Royal Surrey County Hospital NHS Foundation Trust, Guildford, UK); Izhar Bagwan (Royal Surrey County Hospital NHS Foundation Trust, Guildford, UK); Vicki Save (Edinburgh Royal Infirmary, Edinburgh, UK); Richard J E Skipworth (Edinburgh Royal Infirmary, Edinburgh, UK); Ted R Hupp (Edinburgh Royal Infirmary, Edinburgh, UK); Olga Tucker (University Hospitals Birmingham NHS Foundation Trust, Birmingham, UK; Heart of England NHS Foundation Trust, Birmingham, UK); Andrew Beggs (University Hospitals Birmingham NHS Foundation Trust, Birmingham, UK; Institute of Cancer and Genomic Sciences, University of Birmingham, Birmingham, UK); Philippe Taniere (University Hospitals Birmingham NHS Foundation Trust, Birmingham, UK); Sonia Puig (University Hospitals Birmingham NHS Foundation Trust, Birmingham, UK); Jack Owsley (University Hospital Southampton NHS Foundation Trust, Southampton, UK); Hugh Barr (Gloucester Royal Hospital, Gloucester, UK); Neil Shepherd (Gloucester Royal Hospital, Gloucester, UK); Oliver Old (Gloucester Royal Hospital, Gloucester, UK); Jesper Lagergren (Guy's and St Thomas's NHS Foundation Trust, London, UK; Karolinska Institutet, Stockholm, Sweden) James Gossage (Guy's and St Thomas's
NHS Foundation Trust, London, UK; King's College London, London, UK); Andrew Davies (Guy's and St Thomas's NHS Foundation Trust, London, UK; King's College London, London, UK); Fuju Chang (Guy's and St Thomas's NHS Foundation Trust, London, UK; King's College London, London, UK); Janine Zylstra (Guy's and St Thomas's NHS Foundation Trust, London, UK; King's College London, London, UK); Ula Mahadeva (Guy's and St Thomas's NHS Foundation Trust, London, UK); Vicky Goh (King's College London, London, UK); Francesca D Ciccarelli (King's College London, London, UK); Grant Sanders (Plymouth Hospitals NHS Trust, Plymouth, UK); Richard Berrisford (Plymouth Hospitals NHS Trust, Plymouth, UK); Catherine Harden (Plymouth Hospitals NHS Trust, Plymouth, UK); Mike Lewis (Norfolk and Norwich University Hospital NHS Foundation Trust, Norwich, UK); Ed Cheong (Norfolk and Norwich University Hospital NHS Foundation Trust, Norwich, UK); Bhaskar Kumar (Norfolk and Norwich University Hospital NHS Foundation Trust, Norwich, UK); Simon L Parsons (Nottingham University Hospitals NHS Trust, Nottingham, UK); Irshad Soomro (Nottingham University Hospitals NHS Trust, Nottingham, UK); Philip Kaye (Nottingham University Hospitals NHS Trust, Nottingham, UK); John Saunders (Nottingham University Hospitals NHS Trust, Nottingham, UK); Laurence Lovat (University College London, London, UK); Rehan Haidry (University College London, London, UK); Laszlo Igali (Norfolk and Waveney Cellular Pathology Network, Norwich, UK); Michael Scott (Wythenshawe Hospital, Manchester, UK); Sharmila Sothi (University Hospitals Coventry and Warwickshire NHS, Trust, Coventry, UK); Sari Suortamo (University Hospitals Coventry and Warwickshire NHS, Trust, Coventry, UK); Suzy Lishman (Peterborough Hospitals NHS Trust, Peterborough City Hospital, Peterborough, UK); George B Hanna (Department of Surgery and Cancer, Imperial College London, UK); Krishna Moorthy (Department of Surgery and Cancer, Imperial College London, UK); Christopher J Peters (Department of Surgery and Cancer, Imperial College London, UK); Anna Grabowska (Queen's Medical Centre, University of Nottingham, Nottingham, UK); and Helen Coleman (Centre for Cancer Research and Cell Biology, Queen's University Belfast, Northern Ireland, UK).

Contributors RCT, RK and RF contributed to the study concept, design and supervision. RCT had access to all of the data and takes responsibility for data integrity and data analysis. RCT and RK obtained funding for the study. LAK, JKB, MS, AMM, CJS and JP conducted the bioinformatic analysis. All other authors contributed to interpretation of data and critical revision of the manuscript for intellectual content.

Funding This work is funded in part by Belfast Health and Social Care Trust Gastrointestinal Cancer Research Fund, Almac Diagnostics, Medical Research Council, Cancer Research UK, HSC Research and Development Division of the Public Health Agency in Northern Ireland, National Institute for Health Research (NIHR) Cambridge Biomedical Research Centre and Invest Northern Ireland.

Competing interests LAK, AMM, SMW, DPH and RK are employees of Almac Diagnostics and have patent declarations. GEL and CJS are employees of Almac Diagnostics.

\section{Patient consent for publication Not required.}

Provenance and peer review Not commissioned; externally peer reviewed.

Open access This is an open access article distributed in accordance with the Creative Commons Attribution Non Commercial (CC BY-NC 4.0) license, which permits others to distribute, remix, adapt, build upon this work non-commercially, and license their derivative works on different terms, provided the original work is properly cited, appropriate credit is given, any changes made indicated, and the use is non-commercial. See: http://creativecommons.org/licenses/by-nc/4.0/.

\section{REFERENCES}

1 Brown LM, Devesa SS, Chow WH. Incidence of adenocarcinoma of the esophagus among white Americans by sex, stage, and age. J Natl Cancer Inst 2008;100:1184-7.

2 Edgren G, Adami HO, Weiderpass E, et al. A global assessment of the oesophageal adenocarcinoma epidemic. Gut 2013;62:1406-14.

3 Ferlay J, Soerjomataram I, Ervik M, et al. v1.0, Cancer Incidence and Mortality Worldwide: IARC CancerBase No. 11. 2013. Lyon, France: International Agency for Research on Cancer, 2012

4 Cunningham D, Allum WH, Stenning SP, et al. Perioperative chemotherapy versus surgery alone for resectable gastroesophageal cancer. N Engl J Med 2006;355:11-20.

5 Allum WH, Stenning SP, Bancewicz J, et al. Long-term results of a randomized trial of surgery with or without preoperative chemotherapy in esophageal cancer. J Clin Oncol 2009;27:5062-7.

6 van Hagen P, Hulshof MC, van Lanschot JJ, et al. Preoperative chemoradiotherapy for esophageal or junctional cancer. N Engl J Med 2012;366:2074-84.

7 Noble F, Lloyd MA, Turkington R, et al. Multicentre cohort study to define and validate pathological assessment of response to neoadjuvant therapy in oesophagogastric adenocarcinoma. Br J Surg 2017;104:1816-28.

8 Ott K, Weber WA, Lordick F, et al. Metabolic imaging predicts response, survival, and recurrence in adenocarcinomas of the esophagogastric junction. J Clin Oncol 2006;24:4692-8 
9 Lordick F, Ott K, Krause BJ, et al. PET to assess early metabolic response and to guide treatment of adenocarcinoma of the oesophagogastric junction: the MUNICON phase II trial. Lancet Oncol 2007;8:797-805.

10 Wieder HA, Ott K, Lordick F, et al. Prediction of tumor response by FDG-PET: comparison of the accuracy of single and sequential studies in patients with adenocarcinomas of the esophagogastric junction. Eur J Nucl Med Mol Imaging 2007:34:1925-32.

11 Abdel-Latif MM, O'Riordan J, Windle HJ, et al. NF-kappaB activation in esophageal adenocarcinoma: relationship to Barrett's metaplasia, survival, and response to neoadjuvant chemoradiotherapy. Ann Surg 2004;239:491-500.

12 Izzo JG, Correa AM, Wu TT, et al. Pretherapy nuclear factor-kappaB status, chemoradiation resistance, and metastatic progression in esophageal carcinoma. $\mathrm{Mol}$ Cancer Ther 2006;5:2844-50.

13 Izzo JG, Malhotra U, Wu TT, et al. Association of activated transcription factor nuclear factor kappab with chemoradiation resistance and poor outcome in esophageal carcinoma. J Clin Oncol 2006;24:748-54.

14 Gibson MK, Abraham SC, Wu TT, et al. Epidermal growth factor receptor, p53 mutation, and pathological response predict survival in patients with locally advanced esophageal cancer treated with preoperative chemoradiotherapy. Clin Cancer Res 2003;9:6461-8

15 Schneider S, Uchida K, Brabender J, et al. Downregulation of TS, DPD, ERCC1, GST-Pi, EGFR, and HER2 gene expression after neoadjuvant three-modality treatment in patients with esophageal cancer. J Am Coll Surg 2005;200:336-44.

16 Joshi MB, Shirota Y, Danenberg KD, et al. High gene expression of TS1, GSTP1, and ERCC1 are risk factors for survival in patients treated with trimodality therapy for esophageal cancer. Clin Cancer Res 2005;11:2215-21.

17 Warnecke-Eberz U, Metzger R, Miyazono F, et al. High specificity of quantitative excision repair cross-complementing 1 messenger RNA expression for prediction of minor histopathological response to neoadjuvant radiochemotherapy in esophageal cancer. Clin Cancer Res 2004;10:3794-9.

18 Harpole DH, Moore MB, Herndon JE, et al. The prognostic value of molecular marker analysis in patients treated with trimodality therapy for esophageal cancer. Clin Cancer Res 2001;7:562-9.

19 Dulak AM, Stojanov P, Peng S, et al. Exome and whole-genome sequencing of esophageal adenocarcinoma identifies recurrent driver events and mutational complexity. Nat Genet 2013;45:478-86.

20 Weaver JMJ, Ross-Innes CS, Shannon N, et al. Ordering of mutations in preinvasive disease stages of esophageal carcinogenesis. Nat Genet 2014;46:837-43.

21 Ross-Innes CS, Becq J, Warren A, et al. Whole-genome sequencing provides new insights into the clonal architecture of Barrett's esophagus and esophageal adenocarcinoma. Nat Genet 2015;47:1038-46.

22 Secrier M, Li X, de Silva N, et al. Mutational signatures in esophageal adenocarcinoma define etiologically distinct subgroups with therapeutic relevance. Nat Genet 2016;48:1131-41.

23 Luthra R, Wu TT, Luthra MG, et al. Gene expression profiling of localized esophageal carcinomas: association with pathologic response to preoperative chemoradiation. J Clin Oncol 2006;24:259-67.

24 Duong C, Greenawalt DM, Kowalczyk A, et al. Pretreatment gene expression profiles can be used to predict response to neoadjuvant chemoradiotherapy in esophageal cancer. Ann Surg Oncol 2007;14:3602-9.

25 Schauer M, Janssen KP, Rimkus C, et al. Microarray-based response prediction in esophageal adenocarcinoma. Clin Cancer Res 2010;16:330-7.

26 Mulligan JM, Hill LA, Deharo S, et al. Identification and validation of an anthracycline/ cyclophosphamide-based chemotherapy response assay in breast cancer. J Natl Cancer Inst 2014;106:djt335.

27 Parkes EE, Walker SM, Taggart LE, et al. Activation of STING-Dependent innate immune signaling by S-Phase-Specific DNA Damage in Breast Cancer. J Nat/ Cancer Inst 2017;109:djw199.
28 Mandard AM, Dalibard F, Mandard JC, et al. Pathologic assessment of tumor regression after preoperative chemoradiotherapy of esophageal carcinoma. Clinicopathologic correlations. Cancer 1994;73:2680-6.

29 Peters CJ, Rees JR, Hardwick RH, et al. A 4-gene signature predicts survival of patients with resected adenocarcinoma of the esophagus, junction, and gastric cardia. Gastroenterology 2010;139:1995-2004.

30 Kennedy RD, Bylesjo M, Kerr P, et al. Development and independent validation of a prognostic assay for stage II colon cancer using formalin-fixed paraffin-embedded tissue. Journal of Clinical Oncology 2011;29:4620-6.

31 Blayney JK, Cairns L, Li G, et al. Glucose transporter 1 expression as a marker of prognosis in oesophageal adenocarcinoma. Oncotarget 2018;9:18518-28.

32 Ilyas M, Grabsch H, Ellis 10, et al. Guidelines and considerations for conducting experiments using tissue microarrays. Histopathology 2013;62:827-39.

33 Diggs LP, Hsueh EC. Utility of PD-L1 immunohistochemistry assays for predicting PD-1/ PD-L1 inhibitor response. Biomark Res 2017;5:12.

34 Alexandrov LB, Nik-Zainal S, Siu HC, et al. A mutational signature in gastric cancer suggests therapeutic strategies. Nat Commun 2015;6:8683.

35 van der Kaaij RT, Snaebjornsson P, Voncken FE, et al. The prognostic and potentially predictive value of the Laurén classification in oesophageal adenocarcinoma. Eur J Cancer 2017;76:27-35.

36 Alderson D, Cunningham D, Nankivell M, et al. Neoadjuvant cisplatin and fluorouracil versus epirubicin, cisplatin, and capecitabine followed by resection in patients with oesophageal adenocarcinoma (UK MRC OE05): an open-label, randomised phase 3 trial. Lancet Oncol 2017;18:1-12.

37 De Silva N, Schulz L, Paterson A, et al. Molecular effects of Lapatinib in the treatment of HER2 overexpressing oesophago-gastric adenocarcinoma. $\mathrm{Br} J \mathrm{Cancer}$ 2015;113:1305-12.

38 Motoori M, Takemasa I, Yamasaki M, et al. Prediction of the response to chemotherapy in advanced esophageal cancer by gene expression profiling of biopsy samples. Int J Oncol 2010;37:1113-20.

39 Findlay JM, Castro-Giner F, Makino S, et al. Differential clonal evolution in oesophageal cancers in response to neo-adjuvant chemotherapy. Nat Commun 2016;7:11111.

40 Quinn JE, James CR, Stewart GE, et al. BRCA1 mRNA expression levels predict for overall survival in ovarian cancer after chemotherapy. Clin Cancer Res 2007;13:7413-20.

41 Quinn JE, Kennedy RD, Mullan PB, et al. BRCA1 functions as a differential modulator of chemotherapy-induced apoptosis. Cancer Res 2003;63:6221-8.

42 Bass AJ, Thorsson V, Shmulevich I, et al. Comprehensive molecular characterization of gastric adenocarcinoma. Nature 2014;513:202-9.

43 Cancer Genome Atlas Research Network. Integrated genomic characterization of oesophageal carcinoma. Nature 2017;541.

44 Smyth EC, Cafferkey C, Loehr A, et al. Genomic loss of heterozygosity and survival in the REAL3 trial. Oncotarget 2018;9:36654-65.

45 Linger RJ, Kruk PA. BRCA1 16 years later: risk-associated BRCA1 mutations and their functional implications. Febs J 2010;277:3086-96.

46 Esteller M, Silva JM, Dominguez G, et al. Promoter hypermethylation and BRCA1 inactivation in sporadic breast and ovarian tumors. J Nat/ Cancer Inst 2000;92:564-9.

47 Turner N, Tutt A, Ashworth A. Hallmarks of 'BRCAness' in sporadic cancers. Nat Rev Cancer 2004;4:814-9.

48 Mouw KW, Goldberg MS, Konstantinopoulos PA, et al. DNA Damage and repair biomarkers of immunotherapy response. Cancer Discov 2017;7:675-93.

49 Wang H, Hu S, Chen X, et al. cGAS is essential for the antitumor effect of immune checkpoint blockade. Proc Natl Acad Sci U S A 2017;114:1637-42.

50 Zaretsky JM, Garcia-Diaz A, Shin DS, et al. Mutations associated with acquired resistance to PD-1 blockade in Melanoma. N Engl J Med 2016;375:819-29.

51 Daud Al, Loo K, Pauli ML, et al. Tumor immune profiling predicts response to antiPD-1 therapy in human melanoma. J Clin Invest 2016;126:3447-52.

52 Patel SP, Kurzrock R. PD-L1 Expression as a predictive biomarker in cancer immunotherapy. Mol Cancer Ther 2015;14:847-56. 